\title{
UNE NOUUVLLE CARTE LINGUISTIQUE DE LA SUISSE
}

\author{
commentée par Aldo Dami
}

avec une carte en couleurs

La carte des langues parlées en Suisse ou plus exactement par la population suisse, carte que nous devons au labeur intelligent et consciencieux de M. H. FREY, illustre en premier lieu une situation que les étrangers ont souvent peine à comprendre. Beaucoup d'entre eux s'imaginent volontiers, en effet, que les Suisses sont trilingues, voire quadrilingues, alors qu'au contraire les limites de langue, en Sưisse, sont très tranchées, comme elles le sont en généralement en Occident. Il arrive parfois (sur la frontière Vaud-Berne et Valais-Berne, notamment) qu'on ne se comprenne pas d'un chalet à l'autre même lorsqu'ils ne sont distants que de quelques centaines de mètres.

Ce phénomène est très explicable. Dans l'Occident de l'Europe, les domaines linguistiques - à défaut des langues elles-mêmes - sont stabilisés ou à peu près stabilisés depuis des siècles, parce que les formes d'Etat y sont également très anciennes et que les modifications de frontières, depuis 1815 en tout cas, y sont rares ou de faible ampleur. Tout autre est la situation en Orient où, d'une guerre à l'autre, les frontières sont instables par définition, où les langues slaves, en particulier, sont si apparentées qu'il est souvent difficile de tracer leurs limites, et où enfin l'oppression chronique des minorités et plus récemment d'énormes transferts de population ont pour effet de continuelles et parfois très profondes modifications de la situation "ethnique» (le mot "ethnique» étant entendu ici dans son sens populaire, mais erroné, de "linguistique»). En Suisse même, le fait que les deux principales langues latines, le français et l'italien, ne confinent pas entre elles, accentue la "coupure»: entre l'allemand d'une part, le français, l'italien et le rhéto-romanche de l'autre, il n'y a pas de dialecte intermédiaire possible.

Une seconde erreur courante à l'étranger consiste à confondre notre situation d'Etat multilingue avec notre fédéralisme politique, bref à croire que la Suisse a une structure fédéraliste parce qu' elle est multilingue. Rien de plus faux. D'abord c'est un anachronisme: la forme de l'Etat suisse, d'emblée fédérative et de façon plus marquée encore qu'aujourd'hui, date d'une époque où le pays ne parlait que l'allemand; elle est bien antérieure à la conquête des baillages français et italiens. Notre fédéralisme est certes linguistique aussi, en ce sens que toutes les langues parlées dans le pays - et même les autres y bénéficient, sur tout le territoire, de la plus large tolérance, ce qui n'est certes pas le cas ailleurs et pas même en France, en Espagne ou en Italie. Mais si notre fédéralisme n'était que cela, il n'y aurait en Suisse non pas 22 cantons mais 4 , au maximum 5 si l'on distingue encore le ladin du romanche. Or ces deux dialectes rhétoromans, au contraire, sont parlés dans le même canton et, en Suisse, exclusivement là. Les Grisons, de plus, sont trilingues et mêmes quadrilingues; trois autres cantons, Berne, Fribourg et Valais, sont bilingues. Les limites de langue chevauchent donc celles des cantons et ne coincident même avec les limites cantonales que sur de faibles tronçons: partie de la frontière Berne-Soleure entre Elay (Seehof) et Romont; frontière Berne-Neuchâtel si l'on fait exception du cas de Thielle-Wavre dont nous parlerons plus loin; frontière Berne-Vaud sur toute sa longueur; frontière Berne-Valais sur le quart environ de son parcours; frontière entre le Tessin d'une part, le Valais, Uri et les Grisons jusqu'à la source du Rhin postérieur; frontière entre les Grisons d'une part, Uri et Glaris de l'autre.

Ainsi donc, quatre cantons ont deux ou même trois langues officielles, alors qu'il n'y en a qu'une dans les 18 autres cantons, bien que l'allemand soit la langue d'une commune tessinoise, Bosco-Gurin, dont nous parlerons également plus loin. Notre "tolérance» linguistique est donc cantonale avant même d'être fédérale.

Nous n'allons pas tracer ici, sous peine d'accumuler les noms propres, la limite des langues qu'on peut suivre admirablement sur la carte de M. FrEY. Celle-ci nous dispense donć d'entrer dans les détails. Bornons-nous plutôt à quelques considérations générales, pour terminer en signalant quelques cas particuliers parmi les plus intéressants.

On voit du premier coup d'œil que l'allemand, ou plus exactement les dialectes alémanniques: bas-alémane au nord du Jura (Bâle forme ici un groupe avec l'Alsace méridionale et moyenne et le sud du pays de Bade), haut-alémane, subdivisé lui-même en cinq ou six groupes, dans tout le reste de la Suisse allemande sauf Samnaun, occupe de beaucoup la plus grande partie du territoire suisse. Il est parlé, compte tenu des nombreuses et puissantes colonies alémanniques en Suisse romande et italienne, par près de $73 \%$ de la population, autant dire les trois quarts; et du fait de la prolificité supérieure des Suisses-allemands, cette proportion ne cesse de s'accroître: elle n'était 
que de $69 \%$ en 1910 . Nous y reviendrons également en parlant du déplacement des limites linguistiques.

L'exception de Samnaun, seul village de Suisse allemande où l'on ne parle pas le suisse allemand (magnifique question pour un examen de géographie!) s'explique par le fait que cette dernière commune grisonne, à l'extrême frontière orientale de la Suisse, est entièrement séparée du reste de la Suisse allemande par le domaine ladin qui couvre la Basse-Engadine. Gravitant déjà sur le Tyrol, et non plus sur le Vorarlberg qui est encore alémannique, Samnaun parle donc le dialecte bavarois qui, on le sait, couvire toute l'Autriche, l'ex-pays des Sudètes et la Bavière méridionale (à l'exception donc de la Souabe bavaroise, des trois Franconies et du Palatinat).

L'allemand sépare entièrement, sur le sol suisse, le français de l'italien. Les domaines de ces deux langues ne se rejoignent qu'à Issime, sur territoire italien, dans la vallée du Lys qui descend du Mont-Rose.

Les Alémanes en effet, qui avaient leur résidence primitive entre le Main et le Neckar, ont sans cesse poussé obstinément vers le sud. Dès le IIIle siècle après J.-Chr. ils cherchent à forcer le limes romain qui va du Rhin au Danube et qui couvre les "Champs décumates». Ils y réussissent entre le Ve et le Vle siècles. Ils envahissent enfin l'Helvétie elle-même : la Suisse du Nord est germanisée au plus tard vers le milieu du VIe siècle; la Suisse centrale, au IXe et au Xe siècle seulement; Coire a même parlé roman jusqu'au XVe.

Nous ignorons si les Helvètes et les Rhètes romanisés opposèrent une résistance aux Alémanes. Ce que l'on sait, c'est que ceux-ci entrèrent en lutte avec les Burgondes, et que ces derniers furent vaincus. Ce qu'on appelait alors la Haute- ou la Grande-Bourgogne s'étendait à l'est jusqu'à la Reuss; mais des Alémanes en peuplaient déjà la partie orientale. En un premier temps, les Burgondes furent refoulés de la Reuss à l'Aar, y compris l'Oberland; puis - mais cette fois-ci sans notable déplacement de population - ils furent re-germanisés entre l'Aar et la Sarine. La frontière linguistique de l'Aar, qui subsista trois siècles, est prouvée par la toponomastique. Ainsi les Soleurois, une partie des Bernois, et les Fribourgeois de langue allemande, ne sont pas des Alémanes, mais des Burgondes redevenus de langue germanique.

Mais ce n'est pas tout. Après avoir remonté les vallées jusqu'aux Alpes, les Alémanes franchirent la chaîne. Ils en franchirent même deux; car, arrêtés au St-Gothard - seul endroit précisément où il n'y aurait eu qu'un seul col à passer, mais où le défilé de la Reuss était alors infranchissable (d'où aussi la longue persistance du roman dans la vallée d'Urseren, qui dépendit pendant des siècles de l'abbaye de Disentis) - ils débordèrent, plus à l'ouest, non seulement les Alpes bernoises mais les Alpes pennines.

Ce fut d'abord la poussée sur le Haut-Valais, par le Grimsel et peut-être par la Gemmi. Celle-ci déjà a eu, pour la future Suisse du moins, des conséquences capitales, parce que c'est alors que l'élément latin du pays a perdu sa cohésion. Avant cette "coupure», la Romania, en Suisse, était d'un seul tenant: le franco-provençal du Valais se fondant, au gré d'une transition insensible, dans le rhéto-romanche de l'Urseren et du reste de la Rhétie.

La vallée centrale du Rhône fut germanisée bien avant les vallées latérales du versant sud: au XIIe siècle, alors que Sion parlait déjà allemand, Zermatt (Praborgne) était encore roman: situation inverse de l'actuelle. Bien mieux: avant le percement du Lötschberg, qui rapproche à nouveau le Haut-Valais du reste de la Suisse allemande, le recul de l'allemand dans la vallée centrale - voie de communication directe, par la route puis le tunnel du Simplon, entre l'Italie d'une part, la Suisse romande et la France de l'autre - était tel qu'on pouvait prévoir le moment où, par la remontée de l'élément romand vers Brigue déjà trilingue, le domaine germanique se trouverait à son tour coupé en deux le long du Rhône, seules les vallées latérales du sud, précisément, conservant l'idiome alémannique.

Quoi qu'il en soit, on peut admettre qu'à la fin du XIIe siècle, la germanisation du Haut-Valais jusqu'à la Morge est achevée. Mais entre temps, par les cols alors aisément franchissables du Théodule et du Monte Moro, et peut-être même par le Lysjoch, l'allemand déborde de toutes parts le massif du Mont-Rose et s'installe sur les versants méridionaux, donc italiens: à St-Jacques sur l'Evançon (où il s'est éteint), à Gressoney sur le Lys, à Alagna sur la Sesia, à Macugnaga sur l'Anza. Il y subsiste encore de nos jours. Plus au nord, il déborde également les Alpes pennines à Simplon-Village, $Z$ wischbergen et Gondo, en territoire suisse, mais aussi dans le val Formazza, en territoire italien.

Cela même explique le cas, en apparence si curieux, de Bosco-Gurin (officiellement Bosco-Valle Maggia sur la carte). Si cette commune en effet, seule dans le Tessin, est de langue allemande, ce n'est pas parce qu'elle confine à la Suisse allemande - dont elle est très éloignée - mais à... l'Italie allemande.

Il s'est produit là un phénomène qu'on retrouve, sur une plus grande échelle, dans l'ensemble de la vallée d'Aoste, ou, plus loin de nous, en Ruthénie subcarpathique, voire au Népal et au Boutan. Ce ne sont pas, en effet, les chaînes de montagne, même les plus hautes et en apparence les plus infranchissables, qui marquent la limite des langues, mais les défilés qui ferment les vallées. De même que l'Himalaya ou les Carpathes, les Alpes ont été franchies, à peu près partout, par des populations en transhumance, qui poussaient leurs troupeaux. Les Alpes en particulier ne forment presque nulle part une frontière de langue, à l'exception du tronçon Berne-Valais déjà cité, du massif du Gothard pour les raisons également déjà indiquées, et d'un bref trajet de la chaine carnique entre l'allemand et le frioulan. 

Leere Seite

Blank page

Page vide 
Les Français les ont débordées de la mer au Cervin, les Allemands du Cervin au col du Gries, dans les Grisons et le Tyrol du Sud, les Slovènes dans le Frioul lui-même. Et de même que le défilé de Bard, dans la vallée d'Aoste, a formé pendant des siècles une barrière bien plus hermétique que les cols des deux St-Bernard, de la Seigne, de Ferret, de Fenètre, du Collon, de l'Evêque ou du Théodule - expliquant ainsi l'évolution identique du Valais, de la Savoie et de la vallée d'Aoste vers un mème patois français - de même, entre le Val Formazza, peuplé par des Haut-Valaisans d'Ulrichen ou de Binn, et leur colonie avancée de Bosco qui allait les faire se retrouver en Suisse ou plutòt dans ce qui devait devenir un baillage suisse, les cols de Obere et de Untere Furka établissaient des communications plus aisées que la gorge qui sépare Bosco de Cerentino, plus en aval. Et comme, plus au nord, la chaîne principale des Alpes lépontiennes, qui coincide avec la frontière politique, marque à nouveau la limite des langues, il en résulte qu'entre Bosco et le col de San Giacomo on parle italien sur le versant suisse et allemand sur le versant italien.

"On parle» est beaucoup dire. L'italianisation, en effet, marche à grands pas dans ces vallées italiennes de langue allemande. Amorcé déjà grâce au tourisme (tant les hôteliers que leurs hôtes viennent du sud) et par l'industrialisation partielle, ce processus s'est accentué et précipité sous le régime fasciste, qui a tout fait pour faire disparaittre aussi vite que possible, sur tout le territoire italien, les minorités allogènes, allant jusqu'à débaptiser non seulement les localités mais les gens, et jusqu'aux tombes dans les cimetières. A Gressoney, les épitaphes sont en trois langues: allemand (langue locale) pour les vieilles générations, français (langue de l'ensemble de la vallée d'Aoste) pour la génération intermédiaire, italien (langue de l'Etat) depuis lors. A Gressoney, à Alagna, à Macugnaga, dans le Val Formazza, l'allemand, sous le fascisme, ne s'enseignait plus qu'en cachette dans les chalets reculés. Il est en voie de disparition complète dans les trois dernières régions. A Gressoney, cette disparition se trouvera simplement retardée du fait de l'autonomie conquise, depuis la guerre, par la vallée d'Aoste dont dépend la vallée du Lys: l'usage et même l'enseignement du français ayant repris dans la nouvelle région autonome, les autorités de la vallée s'occuperont aussi de rétablir un enseignement partiel de l'allemand dans les écoles des trois communes d'Issime, Gressoney-St-Jean et Gressoney-la-Trinité, et l'on sait que le bilinguisme est aussi réintroduit dans les écoles du Tyrol du Sud. Mais ce ne saurait être le cas à Alagna qui dépend de la province deVercelli,à Macugnaga et dans leVal Formazza, qui dépendent de Novare.

A Bosco, en revanche, on se retrouve sur le sol suisse. Malgré l'ouverture de la route de Cevio et les progrès de l'italianisation, en tout cas du bilinguisme, le germanisme, ici, se trouve protégé par l'école allemande financée par une institution de Zurich. De sorte que lorsque l'italianisation du Val Formazza sera achevée, Bosco deviendra, du moins pour de longues années encore, un véritable ilot linguistique, comme il semble l'être déjà si l'on considère la carte qui se borne au territoire suisse, sans pouvoir faire mention, pas plus ici qu’à Gressoney, des "débordements» linguistiques les plus intéressants, qui sont en même temps les plus révélateurs. On ne peut, soit dit en passant, que le regretter.

Si cette carte ne montre pas non plus, pour la mème raison, la colonisation des "Walser» au Vorarlberg et jusqu'en Bavière (Mittelwald) où l'allemand, grâce à eux, a détrôné le rhéto-romanche parlé jadis de Brigue jusqu'à Munich, mais ne forme pas, et pour cause, une enclave ou en tout cas un "curiosum" linguistique, du moins nous explique-t-elle, par les enclaves allemandes d'Obersaxen, de ValsSafien-Rheinwald et d'Avers, la colonisation de ces mêmes Walser sur le territoire actuellement suisse.

Le domaine du français n'appelle guère d'observations spéciales, à ceci près qu'un linguiste distinguerait ici l'origine franco-provençale, qui est celle de presque toute la Suisse romande, dụ français d'oïl parlé au nord d'une ligne approximative La Chaux-deFonds-Bienne. La plus grande partie du Jura bernois, en effet, est d'origine franque et non burgonde; ses patois s'apparentent au franc-comtois du nord, au bourguignon (qui n'est précisément plus "burgonde»), au lorrain et même au wallon. Tout le reste de la Suisse romande, au contraire, forme ou plutôt formait (car, à l'exception du Valais, de la Gruyère, des Ormonts et de quelques villages du Jorat, le patois y a disparu) un seul groupe avec la Franche-Comté méridionale jusqu'à la Saône, le Lyonnais, la Savoie, le Dauphiné du Nord et la vallée d'Aoste. C'est le domaine du franco-provençal, intermédiaire non seulement entre le français et le provençal comme son nom l'indique, mais aussi entre le français proprement dit et le gallo-italien de la plaine du Pô, ainsi qu'entre le provençal et le romanche qu'il reliait jadis, on l'a vu, lorsque le latin des Alpes était encore d'un seul tenant.

Il n'en reste pas moins que le français de France est en Suisse romande une langue importée et que nous, Romands, nous avons conservé toutes les gaucheries des bilingues. La seule - et capitale - dif- 
férence avec la Suisse allemande, c'est que précisément nous avons conscience de nos «fautes» et qu'en luttant contre le "français fédéral» nous nous efforçons de nous rapprocher du français de Paris, alors que les Suisses-allemands, au contraire, ont non seulement conservé leurs dialectes, mais ne manquent pas une occasion de les cultiver et de souligner les différences qu'il y a entre l'allemand "d'Allemagne " et le suisse-allemand et même l' "allemand suisse", cet allemand fédéral qui leur fait non seulement dire mais écrire "vélo" au lieu de Fahrrad, "billet» au lieu de Fahrkarte, "perron" au lieu de Bahnsteig, etc.

Quant à la limite septentrionale de l'italien, qui confine en Suisse à l'allemand et au rhéto-romanche, elle se confond partiellement avec la crête des Alpes et donc avec la limite politique du canton du Tessin (qui déborde légèrement cette crête aux sources de la Reuss et du Rhin de Medels); puis elle englobe, dans les Grisons, les trois vallées de Mesocco (avec la Calanca), directement contiguë encore au Tessin, de la Bregaglia et du Val Poschiavo, qui ne confinent qu'à l'Italie. Ici encore, l'italien du Bergell dépasse la ligne de partage des eaux à Bivio ou Stalla, dans la haute vallée de la Julia, commune marquée mixte italo-romanche sur la carte, mais dont la langue officielle est l'italien, ainsi qu'au village de Maloja (fraction détachée de la commune de Stampa) qui, situé au bord du lac de Sils, appartient au bassin de l'Inn. Les deux vallées non padanes de l'Italie à la frontière grisonne, la vallée rhénane de Lei (où il n'y a d'ailleurs que des chalets) et celle, danubienne, de Livigno, parlent également italien.

Le romanche de l'Oberland grison (surselvan), du Schyn, du Domleschg et de l'Oberhalbstein, le ladin de l'Engadine et des Dolomites, le frioulan de la Vénétie orientale représentent tout ce qui reste du vaste domaine rhéto-romanche d'autrefois, avec ses deux capitales de Coire et d'Augsburg et qui a du moins laissé, dans le Liechtenstein et le Vorarlberg notamment, de nombreux noms de lieu. Déjà fragmenté en divers groupes totalement séparés les uns des autres, ce latin des Alpes orientales, qui appartient au point de vue linguistique à la Romania occidentale, se trouve entièrement coupé de celle-ci depuis que les dialectes de l'Italie du Nord, en perdant l's du pluriel sous l'influence du toscan, ont rallié le reste de l'italien et par conséquent la Romania orientale.

Dans les Grisons mêmes, le domaine r̦héto-romanche s'est constamment rétréci sous la double poussée de l'allemand et, subsidiairement, de l'italien. La germanisation, ici, n'a pas été seulement "horizontale» par la conquête territoriale et l'installation, en plein cœur de ce domaine, des véritables enclaves linguistiques dont nous avons parlé et qu'on peut très bien observer sur la carte; elle a été aussi "verticale», c'est-à-dire intellectuelle, en pénétrant dans les milieux cultivés jusqu'à rendre peu à peu bilingue toute la population qui avait besoin d'un idiome de grande civilisation et de large audience, et adapté à la vie moderne. Aujourd'hui deux septièmes seulement des habitants des Grisons, soit un Suisse sur cent, ont encore le romanche ou le ladin pour langue maternelle. Le mémorable scrutin du 20 février 1938, qui a élevé (alternativement) l'un et l'autre de ces idiomes au rang de langue nationale, a toutefois contribué à les sauver ou tout au moins à en retarder la disparition.

Il s'agit tout d'abord de sauver le fil ténu qui, dans la région de Bonaduz, relie encore le surselvan au dialecte romanche du Domleschg et qui, sur la carte, apparaît déjà comme coupé, sans doute parce que la commune même de Bonaduz a pour langue officielle l'allemand, alors que la commune voisine de Domat (Ems), qui figure comme bilingue, a réintroduit le romanche. Mais en même temps, l'enclave allemande d'Obersaxen tend à se relier, par Ilanz, à celle de Vals-Safien...

Ceci nous amène à la question des enclaves et des «anomalies». Laissons de côté les noms mêmes de communes tels que Mettemberg (Jura bernois), Wallenried, Oberried (Fribourg) ou inversement Courlevon, Coussiberlé (Fribourg) ou Clavaleyres (Berne) dont la consonance ne répond pas, ou plus, à la langue parlée par la majorité des habitants ou à la langue officielle de la commune. On en trouvera de nombreux exemples, notamment, en Nuithonie, dans cette région qui va de Fribourg à Morat et qui - un coup d'œil sur la carte de M. FREY suffit à le montrer - n'est pas seulement la contrée par excellence des enclaves cantonales et des frontières enchevêtrées mais aussi, comme les Grisons, des complexités linguistiques et même religieuses. On y voit notamment le village de Cressier, fief de G. DE REYNOLD, presqu'entièrement entouré de villages allemands.

C'est là la seule enclave ou quasi-enclave de langue française en Suisse. Le domaine rhéto-romanche pouvant être malgré tout considéré comme encore d'un seul tenant, il n'y a pas d'enclave de cette langue, à moins de considérer tout ce domaine linguistique 
comme un vaste enclave entre l'allemand et l'italien, qui ont, plus à l'est et plus à l'ouest, une frontière commune. (Le ladin d'Engadine se reliait jadis, par Meran et Bozen, à celui des Dolomites; dans le Tyrol, qui disait ladin disait protestant, et qui disait allemand disait catholique - la religion de l'Empire. De sorte que depuis la guerre de Trente Ans, la germanisation du Tyrol a peu à peu refoulé le ladin dans les vallées latérales de la rive gauche de l'Adige.) Bivio, de son côté, ne saurait être considéré comme une enclave italienne, car elle est en contact par le Septimer avec le Val Bregaglia. Il y a néanmoins, en Suisse, une enclave italienne de fait: Naters, que M. FREY ne signale pas sur sa carte parce que la langue officielle de ce faubourg de Brigue est incontestablement l'allemand. Toutefois, la carte qui figurait à l'Exposition nationale de Zurich, en 1939, indiquait dans cette commune une majorité de langue italienne - curieux retour historique si l'on songe que les ouvriers italiens du tunnel du Simplon ont fait souche précisément dans le lieu d'où étaient partis, quatre siècles plus tôt, les colons importés dans l'Ossola par le cardinal SchinNer, et qui germanisèrent momentanément Ornavasso et Rimella.

A part ces quelques cas isolés, les seules enclaves proprement dites c'est à dire vraiment territoriales (une ou plusieurs communes de langue officielle autre que celle qui les entoure) sont en Suisse les enclaves allemandes.

Mettons à part, ici encore, Seehof (Elay) et Schelten (La Scheulte) qui touchent au canton de Soleure, Bosco qui est relié au Valais par le Val Formazza, et faisons quelque réserve, dans le reste du Jura bernois, sur les nombreux îlots linguistiques dessinés par M. FreY. S'il est incontestable, en effet, qu'un assez grand nombre de communes jurassiennes sont aujourd'hui totalement ou partiellement germanisćes en fait, soit par la fondation déjà ancienne d'écoles anabaptistes, soit par l'immigration plus récente de paysans de l'ancien canton, il n'en reste pas moins que leur langue officielle demeure le français, à la seule exception, croyons-nous, de Mont-Tramelan dont le secrétaire communal rédige ses actes en allemand. Ce sont ces faits, précisément, qui sont à l'origine de la nouvelle agitation "séparatiste» du Jura; ils ont fait l'objet des incidents que l'on sait et qui se sont traduits par les récents débats et décisions du Grand Conseil bernois. La force d'assimilation de la langue française est d'ailleurs si grande qu'à la deuxième ou à la troisième génération, ces "îlots», situés trop loin de la frontière linguistique, se romaniseront inévitablement - tout comme dans le reste de la Suisse romande - avec ou sans mesures officielles.

Il n'en va pas de mème d'Evilard, qui reste officiellement (voir la carte) de langue française, mais dont la germanisation a suivi celle de Macolin; de Thielle-Wavre (Neuchâtel) et de Faoug (Vaud), tous trois situés à la limite même et où le recensement de 1941 accuse déjà une majorité de langue allemande. Dans ces deux derniers cas, toutefois, la germanisation ne deviendra jamais officielle puisque les communes en question appartiennent à des cantons unilingues. Cela même pose toutefois le problème du déplacement des limites linguistiques, dont nous parlerons pour terminer. Notons d'emblée, cependant, que la "germanisation» du Jura allant de pair avec la romanisation graduelle de l'Alsace, on a assisté depuis peu, dans ces régions, a un véritable chassé-croisé dû à des circonstances politiques et tendant à faire pivoter la limite linguistique sur la Lucelle...

On peut en dire autant de la germanisation partielle de St-Moritz, Samaden, Pontresina, Zuoz, Ascona, et même de Locarno et Lugano, due à l'hôtellerie et au tourisme. C'est un fait qu'à St-Moritz, et depuis près d'un demi-siècle, on entend davantage l'allemand et même l'anglais que le romanche (mais l'anglais à Montreux?); à Ascona davantage, à Locarno autant d'allemand que d'italien. Mais la langue officielle et celle des écoles demeure la langue autochtone, et dans les Grisons comme au Tessin, une vigoureuse action de sauvetage est en cours, favorisée dans les Grisons par le vote de 1938. Quant à l'italianisation partielle des mêmes localités de la Haute-Engadine, notée par M. FreY, elle ne représente aussi qu'une situation de fait. On pourrait, avec le mème droit, peindre en hachures rouges Genc̀ve, Lausanne et forces localités de Suisse romande où la population de langue allemande atteint parfois 25 à $30 \%$. M. FreY s'est borné, dans ces cas, à l'indiquer dans une carte spéciale dite des "minorités». On y voit dessinée au moyen de cercles concentriques la proportion des "allogènes» dans les villes principales. Mais il aurait fallu aussi, à ce titre, indiquer la forte minorité italienne à Bosco-Gurin, ou même renoncer ça et là, sur la frontic̀re linguistique, à suivre les limites communales pour observer la limite réelle: tels villages fribourgeois comme Barberêche, Cerniat, Charmey, étendent leurs limites bien au-delà de la frontière linguistique; les hameaux orientaux de la commune de Barberêche sont déjà purement allemands, de même les mayens de Cerniat et de Charmey situés sur le versant oriental de la Berra puisqu'aussi bien ces communes touchent le Lac Noir; de mème pour les chalets septentrionaux de la commune valaisanne de Savièse, situés sur la Sarine en amont de Gsteig, etc.

Restent donc bien, linguistiquement parlant, trois ilots véritables, tous trois allemands et tous trois situés dans les Grisons, et dont l'un, précisément et de beaucoup le plus vaste: Vals-Safien-Rheinwald, n'en est même pas un à en juger par la carte 
FREY qui attribue Bonaduz à l'allemand, assurant ainsi une continuité territoriale avec le reste de la Suisse allemande.

Il nous reste à dire deux mots de l'aspect diachronique du problème, autrement dit le déplacement des limites linguistiques dans le temps.

Quand on parle du déplacement d'une limite linguistique, il sied de bien distinguer tout d'abord entre le changement de la population elle-même par l'invasion violente (cas de la conquête alémane entre Reuss et Aar) ou pacifique (cas de la pénétration suisse allemande actuelle) et le changement de langue de la part d'une seule et même population (cas, classique, des Gallo-Romains; cas des Burgondes entre Aar et Sarine qui ont réappris le germanique; cas des Bretons qui se francisent tout en étant plus prolifiques que les Français); d'autre part, entre le déplacement de la limite sur toute sa longueur et le déplacement par rayonnement, c'est-à-dire tout d'abord dans les centres de culture, villes et bourgs, situés près de la limite et parfois même assez loin (cas des villes bretonnes, y compris Brest, qui constituent tout d'abord autant d'îlots français entourés de villages bretons), les campagnes ne suivant le mouvement que plus tard, mais rarement au-delà d'un demi-siècle. C'est ce qui s'est déjà produit, par exemple, lors de la latinisation de la Gaule. Les "vagues» sont parties des diverses villae et plus tard des chefs-lieux de diocèses; comme leurs points d'intersection différaient selon les mots, ces différentes limites, enchevêtrées, constituent encore aujourd'hui des zones intermédiaires entre les dialectes.

En traitant des limites linguistiques, il faudrait donc, dans la plupart des cas, parler non pas de lignes mais de zones, avec, à chaque fois, une majorité dans tel lieu donné. Ainsi dans le secteur nord de la limite en Suisse (Jura bernois), la langue française est pénétrée d'allemand sur un vaste territoire, alors que la réciproque n'est pas vraie, sauf à Bienne où un tiers de la population parle encore français, et partiellement à Granges (extension de l'horlogerie). En général, le français avance actuellement pour des raisons industrielles et l'allemand pour des raisons agricoles (fermiers suisses-allemands du Jura bernois, des cantons de Vaud et de Genève et même de Savoie); mais, à une pareille distance de la limite, le changement de langue n'est plus possible, du moins en Suisse, et l'on voit alors les Suisses allemands s'assimiler rapidement, à telle enseigne qu'en Suisse romande mème, la proportion des habitants parlant le français ne cesse d'augmenter, en chiffres absolus dans le Valais, à Neuchàtel et à Berne, en chiffres relatifs à Genève, Vaud et Fribourg. Dans ce dernier canton, d'ailleurs, les raisons confessionnelles jouent un rôle, parce que la grande majorité du canton est catholique; or, la partie allemande correspond sensiblement à la partie réformée. Plus au sud encore (frontière Vaud-Berne et Valais-Berne), la limite est nette parce qu'elle suit en général la crête des montagnes, et parce que les trois cantons situés à l'ouest de cette limite, étant totalement ou en majorité romands, exercent une influence française.

On peut donc dire, en résumé, que l'influence de l'allemand est plus forte dans le nord que dans le sud, et que d'autre part la limite est plus tranchée dans le sud (Alpes) que dans le nord (Plateau et Jura).

Ainsi le parler du Val de Ruz et même de la Chaux-de-Fonds (résidu de la domination prussienne?) est beaucoup plus teinté d'allemand que celui de la ville de Fribourg située pourtant à la limite, et il n'y a pratiquement pas de mots allemands dans le patois gruyérien qui confine lui aussi à l'allemand parlé même dans un village du district (Jaun-Bellegarde). Dans la plaine au contraire, et surtout dans le canton de Berne en majorité allemand, l'influence de l'allemand est considérable; nous l'avons vu à propos du Jura. A Delémont-ville, la pénétration allemande a été très forte au début du sic̀cle, puis lors du percement du Lœtschberg, à cause de l'importance ferroviaire de cette localité, peuplée alors de cheminots alémaniques; elle est moindre depuis que Delémont a été rattachéa l'arrondissementC.F.F.de Lausanne.

En Suisse allemande, le dialecte est parlé dans toutes les classes de la population, y compris dans les milieux les plus cultivés; il a mème une forme écrite; d'autre part le français y est devenu peu à peu une langue seconde, de sorte qu'un grand nombre de Suisses allemands sont pratiquement trilingues. Tandis qu'en Suisse romande, nous l'avons vu, les patois ont en grande partie disparu, et les villes ne connaissent que le français: Nous ne sommes «bilingues» que par les résidus du franco-provençal (dans le vocabulaire) et par l'influence de l'allemand (surtout sur la syntaxe).

En Romandie, c'est la Réforme surtout qui a apporté le français littéraire, par l'intermédiaire de la littérature huguenote: les villes et les cantons protestants ont été francisés bien avant les autres: Genève dès 1750 , Lausanne et Neuchâtel vers 1800 , alors que, nous l'avons vu également, le francoprovençal n'est plus guère vivace que dans les montagnes catholiques de Fribourg et du Valais. Ces patois sont d'ailleurs beaucoup plus différenciés que dans une région de mème grandeur en France: au sein d'un mème canton, le val d'Illiez ne comprend pas le val d'Anniviers. 
Depuis plus d'un siècle, l'allemand est en recul au Valais: la limite qui, au XVIIIe siècle, passait encore à l'ouest de Sion, passe maintenant à l'est de Sierre. De 1475 à 1798, le Bas-Valais, conquis sur la Savoie, a été sujet du Haut; et la résidence des évêques haut-valaisans était à Sion. Mais Sion a perdu peu à peu son caractère germanique; alors qu'en 1860 l'allemand y était encore parlé par les trois quarts de la population (comme enclave, il est vrai, avec Sierre et Bramois), en 1900 cette proportion n'était plus que d'un quart; elle est infime aujourd'hui. Or, un canton tend toujours à adopter la langue de son chef-lieu. Dès 1840 la constitution valaisanne a imposé le bilinguisme, et aujourd'hui les trois cinquièmes environ de la population ne parlent que le français.

Le cas du canton de Fribourg est un peu différent. Là, la limite des langues n'accuse pas un déplacement à sens unique: elle a fluctué au gré des circonstances historiques. Ainsi Morat était encore français au XVe siècle, à l'époque de Charles le Téméraire; Marly, au contraire, était encore allemand au XVIIIe. Quant à Fribourg même, fondée par des princes alémaniques, elle est vite rattachée à l'évêché de Lausanne, et au XIIIe siècle on y prêche en français. Au XVe siècle, retour offensif de l'allemand du fait de l'alliance avec les Confédérés (épisode de Nicolas de Flue). Mais ensuite, au XVIIe et an XVIIITe siècles, toute l'Europe assiste à un vif rayonnement du français, et en 1830, dix ans avant le Valais, le français devient la langue officielle du canton de Fribourg tout entier: depuis lors, en dépit du bilinguisme, le français n'a fait qu'y gagner du terrain. Nous voici donc au quatrième va-et-vient. Plus au nord, le Seeland, d'abord bilingue à l'ouest de l'ancien lit de l'Aar, a été totalement germanisé jusqu'à la Thièle, c'est-a-dire jusqu'à la frontière neuchâteloise, que l'allemand déborde déjà, on l'a vu, sur un point. Plus loin encore, l'allemand a conquis la rive gauche du lac de Bienne, n'y laissant au français que le district de Neuveville.

Si l'on envisage donc dans leur ensemble les changements de la limite du français et de l'allemand en Suisse, on y distinguera cinq grandes périodes au moins: $1^{\circ}$ la fondation, à la limite, de villes par les ducs alémaniques; $2^{\circ}$ la première poussée du français amenée par la domination savoyarde sur toute la future Suisse romande ou à peu près; $3^{\circ}$ après les guerres de Bourgogne, et surtout après la Réforme, la seconde avance de l'allemand due à l'extension de la puissance bernoise (le Gessenay se germanise alors complètement et Bienne partiellement; Morat devient un centre réformé); $4^{\circ}$ à partir du XVIIIe siècle (rayonnement du français), de la Révolution et de l'Empire (conquêtes françaises), la seconde poussée du français sur presque toute la ligne; à ce moment, la cause semble entendue parce que les patois français étant déjà en voie d'extinction, la lutte ne se déroule plus qu'entre les dialectes alémaniques et la langue française, plus fine; $5^{\circ}$ mais, de nos jours, l'allemand reprend sa marche en avant, surtout dans le nord (Seeland et Jura) à la fois grâce à l'influence de la capitale du canton devenue en outre, dès 1848 , capitale fédérale, et grâce à la plus grande prolificité des Suisses allemands. Il faut bien parler de prolificité, en effet, si l'on considère qu'en quarante ans la proportion des Suisses parlant allemand a passé de $69 \%$ à $72,6 \%$, tandis que celle des Suisses parlant français reculait de $22 \%$ à 19,4\%. Comme la limite des deux langues ne s'est presque pas modifiée pendant ce temps, en tout cas pas dans une pareille mesure, c'est donc bien que la population suisse allemande s'accroît davantage, autrement dit que sur un territoire donné, il y a accroissement plus fort de la densité d'un côté que de l'autre.

\section{EINE NEUE SPRACHENKARTE DER SCHWEIZ}

Der Kommentar zur neuen Sprachenkarte der Schweiz von H. FrEY klärt zunächst einige im Ausland geläufige Irrtümer über den schweizerischen Föderalismus. Sodann diskutiert er, statt die Sprachgrenze im gesamten zu untersuchen, besonders einige ihrer bedeutsamern Partien, die durch das Vordringen des alemannischen Bereichs auf italienisches (Gressoney, Formazza usw.), tessinisches (BoscoGurin) und welsches Gebiet (Faoug, Tielle-Wavre) und durch die Walserkolonisation im rätoromanischen Bereich bedingt sind. Sodann skizziert er die Germanisation des Juras. Abschließend erfolgen historische Betrachtungen über die Sprachgrenzverschiebungen in der Schweiz.

\section{UNA NUOVA CARTA LINGUISTICA DELLA SVIZZERA}

Le spiegazioni della nuova carta linguistica della Svizzera del dott. H. Frey delucidano dapprima alcuni errori, comuni all'estero, sul federalismo svizzero. Senza entrare particolarmente nei dettagli dei confini linguistici, l'autore discute poi casi speciali del loro andamento in conseguenza all'infiltrazione dell'elemento alemannico in territorio italiano (Gressoney, Formazza, ecc.), ticinese (Bosco-Gurin) e svizzero-francese (Faoug, Tielle-Wavre), alla colonizzazione dei Walser nella regione reto-romanica e infine alla germanizzazione del Giura. Concludendo seguono considerazioni storichi sugli spostamenti dei confini linguistici nella Svizzera. 